\title{
Incorporating Sweeps and Ejections into Lagrangian Stochastic Models of Spore Trajectories Within Plant Canopy Turbulence: Modeled Contact Distributions Are Heavy-Tailed
}

\author{
A. M. Reynolds
}

Rothamsted Research, Harpenden, Hertfordshire, AL5 2JQ, UK.

Accepted for publication 26 June 2012.

\begin{abstract}
Reynolds, A. M. 2012. Incorporating sweeps and ejections into Lagrangian stochastic models of spore trajectories within plant canopy turbulence: Modeled contact distributions are heavy-tailed. Phytopathology 102:1026-1033.

The turbulent dispersal of fungal spores within plant canopies is very different from that within atmospheric boundary-layers and closely analogous to dispersal within turbulent mixing-layers. The process is dominated by the presence of large coherent flow structures, highvelocity downdrafts (sweeps) and updrafts (ejections), that punctuate otherwise quiescent flow. Turbulent dispersion within plant canopies is best predicted by Lagrangian stochastic (particle-tracking) models because other approaches (e.g., diffusion models and similarity theory) are either inappropriate or invalid. Nonetheless, attempts to construct such models have not been wholly successful. Accounting for sweeps and ejections has substantially worsened rather than improved model

tunnel study of scalar dispersion within plant canopy turbulence. Equally good agreement with this data is obtained using Thomson's (1987) Gaussian model. This bolsters confidence in the application of this simple model to the prediction of spore dispersal within plant canopy turbulence. Contact distributions-the probability distribution function for the distance of viable fungal spore movement until deposition - are predicted to have "heavy" inverse power-law tails. It is known that heavy-tailed contact distributions also characterize the dispersal of spores which pass through the canopy turbulence and enter into the overlying atmospheric boundary-layer. Plant disease epidemics due to the airborne dispersal of fungal spores are therefore predicted to develop as accelerating waves over a vast range of scales-from the within field scale to intercontinental scales. This prediction is consistent with recent analyses of field and historical data for rusts in wheat. Such plant disease epidemics are shown to be governed by space-fractional diffusion equations and by Lévy flights.
\end{abstract} agreement with experimental dispersion data. Here we show how this long-standing difficulty with the formulation of Lagrangian stochastic models can be overcome. The new model is shown to be in good agreement with data from a carefully controlled, well-documented wind-
Additional keywords: dispersive waves, fractional diffusion, infestations, plant disease epidemics.
Zadoks (48) identified the spatiotemporal dynamics of epidemic expansion of plant diseases from foci as being one of the key controversies in plant epidemiology in the 20th century. It is well known that the dynamics of plant disease epidemics are very sensitive to the functional form of the contact distribution-the probability distribution function for the distance of viable fungal spore movement until deposition. Plant disease epidemics are expected to take the form of a constant-velocity travelling wave when the contact distribution has an exponential tail and accelerate over time as a "dispersive" wave when the contact distribution has a "heavy" inverse power-law tail that cannot be bound exponentially $(11,15,21,22,29)$. As a consequence, local sources dominant disease dynamics when the contact distribution has an exponential tail and long-distance dispersal dominates when the contact distribution has a heavy tail (39). The latter can give rise to clustering of new foci of infection and disease beyond the original source $(10,24,38,47)$. Identifying the functional form of the contact distribution is therefore of considerable practical importance when examining the potential effectiveness of disease management strategies, as these are dependent on an accurate evaluation of 'immigrant' inoculum coming into a managed area (2).

Corresponding author: A. M. Reynolds;

E-mail address: andy.reynolds@ rothamsted.ac.uk

http://dx.doi.org/10.1094/PHYTO-01-12-0002

(c) 2012 The American Phytopathological Society
Recent theoretical studies suggest that exponentials and inverse power laws are not competing candidate forms of the tails of contact distributions but are instead representative of different atmospheric conditions (37). Using state-of-the-art Lagrangian stochastic (particle tracking) (LS) models, Reynolds (37) predicted that exponential tails are representative of strongly convective conditions whilst heavy-tails are representative of convective boundary-layers with some wind shear and stable boundarylayers.

Here we extend this line of enquiry by simulating spore dispersal within plant canopy turbulence, i.e., turbulence within and up to several canopy heights above a plant canopy. Plant canopy turbulence is very different in character from turbulence within atmospheric boundary-layers. This is because momentum absorption by the canopy results in an aerodynamically-unstable inflected mean velocity profile. This instability leads to the energetic generation large-scale, high-velocity downdrafts (sweeps) and updrafts (ejections) that punctuate otherwise quiescent flow and thereby make the dominant contribution to turbulent transport into and out of plant canopies $(16,35)$. The resulting turbulence is inhomogeneous and highly non-Gaussian (Fig. 1) and is analogous to that found in "mixing layers" (35).

Turbulent dispersal within canopy flows is best predicted by LS models because other approaches (e.g., diffusion models and similarity theory) are either inappropriate or invalid. Nonetheless, attempts to construct such models have not been wholly successful. Accounting for sweeps and ejections has substantially worsened rather than improved model agreement with experi- 
mental dispersion data (17). Here we show how this long-standing difficulty with the formulation of LS models can be overcome and devise a new LS model that is in close agreement with experimental data from a controlled wind-tunnel study of scalar dispersion within plant canopy turbulence (23). Model predictions for contact distributions are shown to be heavy-tailed.

This prediction finds support in the empirical studies $(5,12,30)$. Cowger et al. (12) and Mundt et al. (30) reported that the windseasonal spread of wheat stripe rust (caused by Puccinia striiformis on wheat Triticum aestivum) at field scales $(<100 \mathrm{~m})$ is consistent with dispersive wave dynamics and so with an inverse power-law contact distribution. Aylor et al. (5) used Rotorods and unmanned aerial vehicles to quantify the aerial dispersal of Phytophthora infestans sporangia from an area source of diseased plants in a potato canopy. Although most sporangia were deposited within a few meters of their source, some sporangia were found at downwind distances of $500 \mathrm{~m}$ from the source.

It is then shown how heavy-tailed contact distributions lead to plant disease epidemic dynamics that are governed by spacefractional diffusion equations and by Lévy flight movement patterns. This provides new opportunities for understanding and predicting plant disease epidemics because fractional diffusion equations and Lévy flights have been studied intensively in other contexts (28).
A
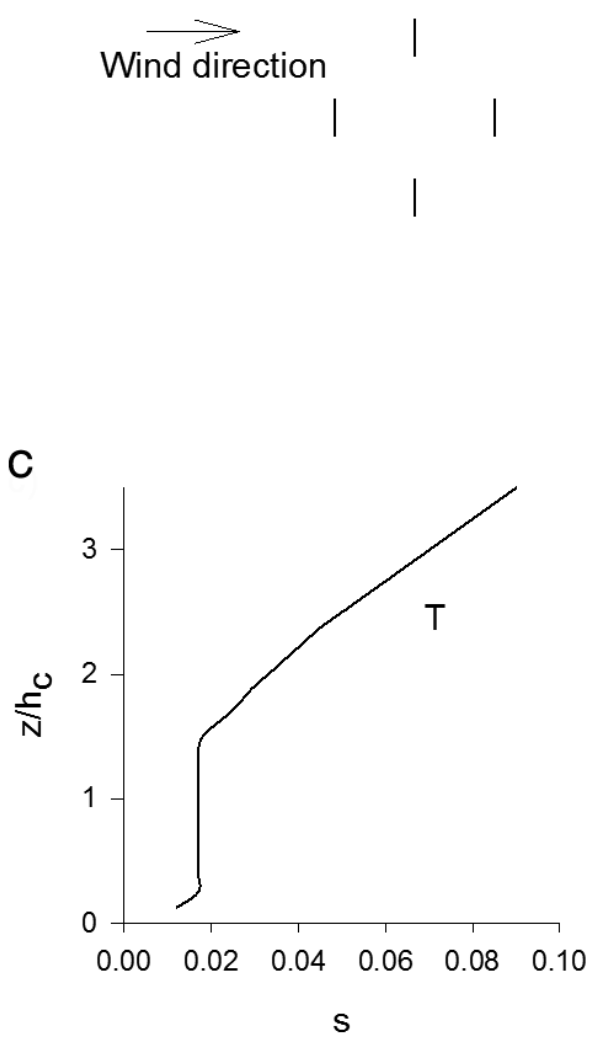

E

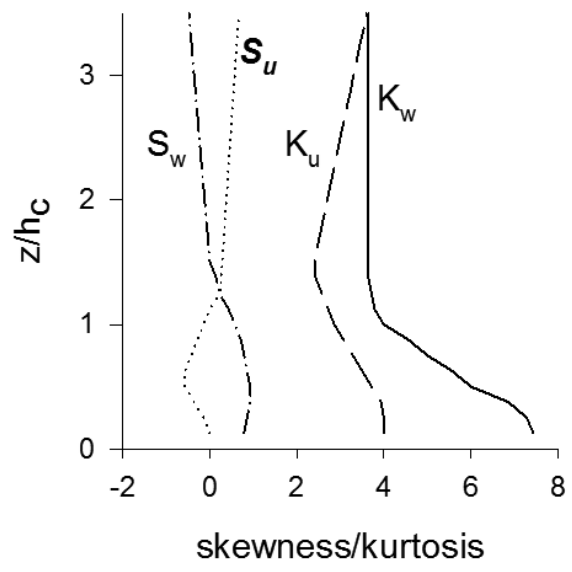

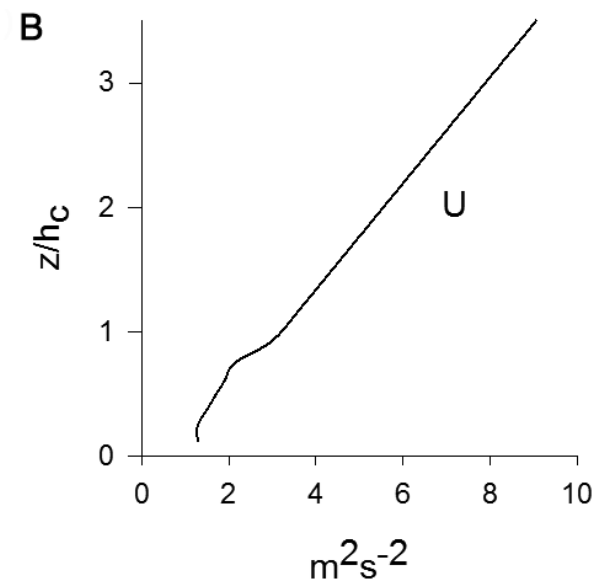

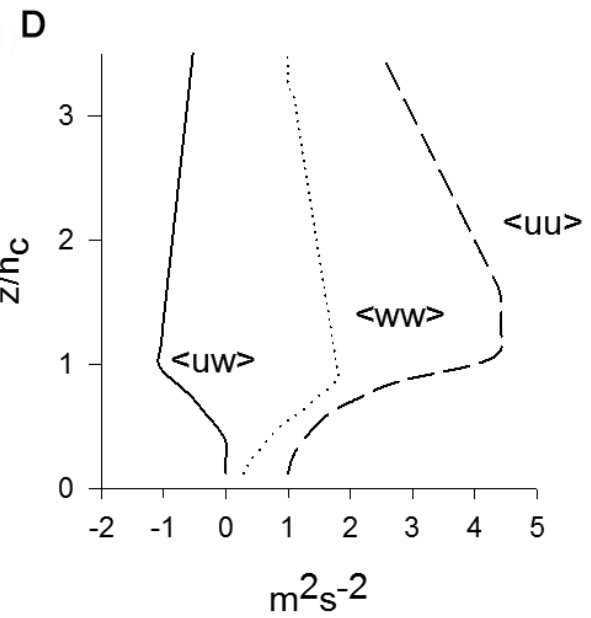




\section{THEORY AND APPROACHES}

LS modeling of turbulent dispersion within plant canopies: Accounting for sweeps and ejections. LS models for the simulation of spore (tracer-particle) trajectories in high Reynolds number turbulence take the form of stochastic differential equations (41)

$$
\begin{gathered}
d x_{i}=\left(U_{i}+u_{i}\right) d t \\
d u_{i}=a_{i}(\underline{u}, \underline{x}, t) d t+b(\underline{x}) d \xi_{i}
\end{gathered}
$$

where $\underline{x}$ is the position of the tracer-particle, $\underline{U}$ and $\underline{u}$ are the mean and fluctuating components of the tracer-particle's velocity, and $d \xi$ is an incremental Wiener processes with zero mean and correlation property $\left\langle d \xi_{i}(t) d \xi_{j}(t+\tau)\right\rangle=\delta_{i j} \delta(\tau) d t$. The subscripts denote Cartesian coordinates and the underscores denote Cartesian vectors. The Markovian assumption is justifiable in the limit of large Reynolds numbers because in this limit the Lagrangian acceleration autocorrelation function approaches a deltafunction, corresponding to an uncorrelated component of Lagrangian acceleration and hence to a Markovian process. The constant $b(x)$ can be determined Kolmogorov similarity theory for turbulence in the inertial subrange. According to this theory, the Lagrangian velocity structure function $\left\langle d u_{i} d u_{j}\right\rangle=\delta_{i j} C_{0} \varepsilon(\underline{x}) d t$ where $C_{0}$ is the Kolmogorov constant and $\varepsilon$ is the mean rate of turbulent dissipation divided by density. This constraint requires that $b^{2}(\underline{x})=C_{0} \varepsilon(\underline{x})$. This specification has found wide spread usage in applications of LS models to the simulation of dispersion in atmospheric boundary-layers. Outcomes of Kolmogorov similarity theory are, however, emphatically violated by most measurements made within plant canopies because plant structures remove energy from large turbulent eddies and diverts it into finer scales, where it is rapidly dissipated, thereby 'short cutting' the inertial eddy-cascade (16). Here, following Flesch and Wilson (17) and others, $b(\underline{x})^{2}$ is here approximated by $2 \sigma_{w}^{2}(\underline{x}) / T(\underline{x})$, where $\sigma_{w}^{2}(\underline{x})$ is the mean-squared fluctuations in the vertical component of velocity and $T(\underline{x})$ is the Lagrangian correlation timescale.

The functional form of $a_{i}(\underline{u}, \underline{x}, t)$ can be constrained (but in general not determined uniquely) by invoking Thomson's "wellmixed condition" (41). Thomson's well-mixed condition currently constitutes the most rigorous framework for the formulation of LS models of tracer-particle motions in high Reynolds number turbulence. It is equivalent to, or more stringent than, all other criteria which have so far been identified as distinguishing between well and poorly formulated models. Thomson's wellmixed condition states that: If at time $t=t_{0}$ the joint distribution of tracer-particle positions and velocities, $P(\underline{u}, \underline{x}, t)$, is proportional to the Eulerian joint distribution of positions and velocities, $P_{E}(\underline{u}, \underline{x}, t)$, then at all later times $t>t_{0} P(\underline{u}, \underline{x}, t)$ must remain proportional to $P_{E}(\underline{u}, \underline{x}, t)$. Mathematically, Thomson's well-mixed condition requires that the model (1) be a solution of the FokkerPlanck equation,

$$
\frac{\partial P_{E}}{\partial t}+\left(U_{i}+u_{i}\right) \frac{\partial P_{E}}{\partial x_{i}}=-\frac{\partial}{\partial u_{i}}\left(a_{i} P_{E}\right)+\frac{b^{2}}{2} \frac{\partial^{2} P_{E}}{\partial u_{i}^{2}}
$$

Flesch and Wilson (17) were the first to invoke Thomson's well mixed condition (41) to construct a two-dimensional LS model that was exactly comparable with measurements of the first four moments of the turbulent velocity distribution made within and just above a plant canopy. Other moments were generally not correct. Third- and fourth-moments do, however, encapsulate key characteristics of sweeps and ejections (16,35). A two-dimensional model allows for an accurate treatment of stream-wise dispersion which is necessary within plant canopies where the horizontal turbulent intensity is large (23). Model agreement with experimental dispersion data (23) was, however, substantially worse than that obtained by a LS model which neglected mea- surements of third- and fourth-order moments and instead assumed that velocities are Gaussian distributed; a Gaussian distribution is completely characterized by its first- and secondorder moments. This shortcoming cannot be attributed to inadequacies in the parameterization of the flow statistics as these statistics were precisely documented (43). Flesch and Wilson (17) suggested that the poor model performance was, instead, due to inaccuracies in the formulation of the modeled velocity distribution. Ambiguities in the specification of this key component in the model arise because the turbulent velocity distribution is not determined uniquely by measurements of its first four moments. Flesch and Wilson (17) utilized a linear combination of two multivariate Gaussian distributions. The suggestion of Flesch and Wilson (17) found in support in the study of Reynolds (36) who showed that an LS model satisfying the well-mixed condition for the maximum-missing-information distribution performed well. The maximum-missing-information distribution maximizes the uncertainty associated with the missing information contained in fifth- and higher-order moments and so is the least biased choice for the velocity distribution.

The results of direct numerical simulations of homogeneous turbulence subsequently revealed that conditional mean Lagrangian accelerations, the focal point of LS modeling, have a quadratic dependency on Lagrangian velocity, at least approximately (6). LS models satisfying the well-mixed condition for Gaussian turbulence have this quadratic-form whilst the models of Flesch and Wilson (17) and Reynolds (36) do not. This suggests that model of Reynolds (36) although successful is inappropriately formulated, and perhaps unreliable.

Here we overcome this difficulty by devising a two-dimensional LS model that is both quadratic in velocity and compatible with prescribed third- and fourth-order moments of the turbulent velocity distribution. This is done following the approach of Franzese et al. (18) who showed that a one-dimensional LS model, quadratic in velocity, can predict accurately turbulent dispersion within convective boundary-layers. Dispersion within convective boundary-layers, like that within plant canopy turbulence, is dominated by the presence of updrafts and downdrafts that produce non-Gaussian velocity statistics. The approach of Franzese et al. (18) has the advantage over Thomson's well-mixed condition (41) of computational efficiency due to algebraic simplicity and of utilizing turbulence statistics up to fourth order without the need to assume any predefined form of turbulent velocity distribution. Satisfaction of the second law thermodynamics is, however, no longer guaranteed as the models could predict wrongly that uniformly distributed particles do not remain uniformly distributed at all later times (33). Compliance with this physical requirement must be tested postea in numerical simulations.

When LS models have a quadratic dependency on velocity

$$
a_{i}(\underline{u}, \underline{x})=\alpha_{i}(\underline{x})+\beta_{i j}(\underline{x}) u_{j}+\gamma_{i j k}(\underline{x}) u_{j} u_{k}
$$

A system of equations that determine the model parameters, $\alpha_{i}(\underline{x}), \quad \beta_{i j}(\underline{x})$, and $\gamma_{i j k}(\underline{x})$, can be obtained by multiplying the Fokker-Planck equation 2 successively by integer powers of $u_{i}$ and then integrating over velocity. For statistical stationary turbulence which is inhomogeneous in only the vertical direction, as is typically the case for plant canopy turbulence, this procedure yields

$$
\begin{gathered}
\frac{\partial}{\partial z}\left\langle u_{3}^{R+1} u_{1}^{S}\right\rangle-S\left[\alpha_{1}\left\langle u_{3}^{R} u_{1}^{S-1}\right\rangle+\beta_{1 j}\left\langle u_{3}^{R} u_{1}^{S-1} u_{j}\right\rangle+\gamma_{1 k j}\left\langle u_{3}^{R} u_{1}^{S-1} u_{j} u_{k}\right\rangle\right] \\
-R\left[\alpha_{3}\left\langle u_{3}^{R-1} u_{1}^{S}\right\rangle+\beta_{3 j}\left\langle u_{3}^{R-1} u_{1}^{S} u_{j}\right\rangle+\gamma_{3 j k}\left\langle u_{3}^{R-1} u_{1}^{S} u_{j} u_{k}\right\rangle\right] \\
-\frac{C_{0} \varepsilon}{2}\left[S(S-1)\left\langle u_{3}^{R} u_{1}^{S-2}\right\rangle+R(R-1)\left\langle u_{3}^{R-2} u_{1}^{S}\right\rangle\right]=0
\end{gathered}
$$


where the subscripts 1 and 3 denote the stream-wise and vertical directions. These 9 equations can constrain but not determine uniquely the 12 model parameters when the first four moments of the Eulerian-velocity distribution are known. To make further progress a closure hypothesis is required. Here the system of equations 4 was closed using by setting $\beta_{12}=\beta_{21}$ and $\gamma_{111}=\gamma_{311}=0$. This closure has the advantage over other possible closures of ensuring that the model, Equations 1 and 3, reduces to Thomson's model (41) when the turbulence becomes Gaussian (as happens well above a canopy) or is taken to be Gaussian. Different models produce different predictions for turbulent dispersion, with Thomson's model being in closest agreement with experimental dispersal data (36).

Simulating scalar dispersion within a model plant canopy. Model predictions for scalar dispersion will be compared with the wind-tunnel study of Legg et al. (23) in which heat was released as a passive scalar from line source into a model plant canopy. Despite their age, these experiments remain the most detailed study of dispersal within canopy turbulence. The model canopy was formed from aluminum strips, each $10 \mathrm{~mm}$ wide, $1 \mathrm{~mm}$ thick, and $h_{c}=60 \mathrm{~mm}$ tall, arranged in a regular diamond pattern with $60 \mathrm{~mm}$ crosswind and $44 \mathrm{~mm}$ stream-wise spacing, as shown in Figure 1A. The line source was perpendicular to the mean flow direction and located at a height $h_{s}=0.85 h_{c}$.

Turbulent velocity statistics required as model inputs were taken from Raupach et al. (34) as reported on by Flesch and Wilson (17), and as shown in Figure 1B and C. These turbulent velocity statistics closely resemble those found in plant canopies (16). Flesch and Wilson (17) reported on turbulent velocity statistics for heights up to about $210 \mathrm{~mm}$. Above this height, turbulent velocity statistics are taken to be stationary and homogeneous. At the ground-level, turbulence statistics are closely Gaussian and so perfect reflection can be used to implement an unattainable boundary condition that is compatible with the local velocity distribution (42). Measurements of the first four Eulerian moments of velocity did not encompass the mixed fourth-order moments $(\langle u u u w\rangle$, etc.) required for the solution of equation 4. Here the mixed fourth-order moments are prescribed by the Millionshchikov approximation

$$
\left\langle u_{i} u_{j} u_{k} u_{l}\right\rangle=\left\langle u_{i} u_{j}\right\rangle\left\langle u_{k} u_{l}\right\rangle+\left\langle u_{i} u_{k}\right\rangle\left\langle u_{j} u_{l}\right\rangle+\left\langle u_{i} u_{l}\right\rangle\left\langle u_{j} u_{k}\right\rangle
$$

Initial velocities were drawn randomly from a bi-Gaussian distribution whose first four moments correspond with experimental measurements made at the source height. The time-step of numerical integration was taken to be $\Delta t=0.001 \min \left(T, \sigma_{i} /\left|a_{i}\right|\right)$ which is sufficient to ensure that inhomogeneities in the flow are properly resolved. Model predictions did not change significantly when smaller values of $\Delta t$ were used.

Statistical stationary predictions for profiles of mean temperature and temperature fluxes were obtained by ensemble averaging over $10^{5}$ simulated tracer-particle trajectories.

The tail of a contact distribution characterizes the long distance dispersal of spores (as well as minute aerially dispersed pests, seeds and pollens) that have been lifted above the top of the canopy by ejections (32). Model predictions for these tails were obtained by simulating the trajectories of many spores from the aforementioned line source and then recording the distances that they travelled after first exiting the canopy. It was assumed these spores were deposited at the points where they first reentered the canopy.

Discriminating between inverse power law and exponential tails. The Akaike information criterion (AIC) was used to test whether the simulation data from the LS model provided more evidence for spore flight distances coming from distributions with inverse power-law tails or from distributions with exponential tails. The Akaike weight, $w$, for the inverse power-law tail can be considered as the weight of evidence in favor of the distribution with the inverse power-law tail being the better model of the simulation data. It ranges between 0 (no support) and 1 (complete support). The goodness-of-fit of the better model distribution was quantified using the G-test. Plots of the survival function (the complement of the cumulative distribution function) are also used to examine the form of the tails and to determine the extent of power-law scaling when present: an approach that is more reliable than probability density function plots (46). To construct the survival function, the simulation data $\left\{l_{i}\right\}$ is first ranked from largest to smallest $\{i=1 \ldots n\}$. The probability that a flight length is greater than or equal to $l_{i}$ (the survival function) is then estimated as $i / n$.

\section{RESULTS}

Comparison of predicted and measured scalar concentrations. Model agreement with the dispersion data of Legg et al. (23) is good except for close to the ground (Fig. 2). The underprediction of mean temperatures close to the ground may be attributed to experimental complications. The plume centroid at the source was measured by Legg et al. (23) and found to lie significantly below the source height. Legg et al. (23) suggested that this was due to the existence of an organized re-circulating flow (caused by canopy elements) which resulted in an upstream heat transfer in the lower canopy. If this is the case then a temperature underprediction near the ground would be unavoidable. At long times predicted temperature profiles are nearly uniform. This was not guaranteed a priori but is required on physical grounds (33).

Predicted temperature fluxes are also in good agreement with experiment (Fig. 2). At most heights the predictions are not statistically different from the measurements and a large fetches the model predicts correctly that temperature fluxes approach zero.

Predicted mean temperatures and temperature fluxes obtained using the new model do not differ significantly from those obtained using Thomson's model (41) (Fig. 2).

Predicted contact distributions. The model prediction for the tail of the contact distribution is very well represented by inverse power law $(w=1.00, G=203, \mathrm{df}=235)$ for distances ranging between about 5 and 500 canopy heights (Fig. 3). The maximum likelihood estimate for $\mu=1.00$. The simulation data are seen to be poorly represented by the best-fit exponential.

Contact distributions, fractional diffusion, and epidemic dynamics. When the density of healthy host plants is not limiting, spatiotemporal disease dynamics depend only on the contact distribution and are governed by

$$
p_{n}(x)=R_{0} \int_{-\infty}^{\infty} p_{n-1}(s) P(x-s) d s
$$

where $p_{n}(x)$ is the disease density at position $x$ after $n$ generations (cycles), $R_{0}$ is the basic reproduction number, and $P(x)$ is the contact distribution - the probability distribution function for the distance of fungal spore movement until deposition (26). Here it is shown that equation 6 is equivalent to a fractional diffusion equation of order $1 / 2, \partial p_{t} / \partial t=D\left(\partial^{1 / 2} p_{t} / \partial x^{1 / 2}\right)$, when the contact distribution has a $-3 / 2$ inverse power-law tail, i.e., when $P(x) \sim x^{-3 / 2}$ for large distances. More general cases are presented later.

The derivation begins by noting that equation 6 with $R_{0}=1$ is equivalent to

$$
\hat{p}_{n}(k)=\hat{P}(k) \hat{p}_{n-1}(k)
$$

where

$$
\begin{aligned}
& \hat{p}_{n}(k)=\int_{0}^{\infty} p_{n}(x) e^{i k x} d x \\
& \hat{P}(k)=\int_{0}^{\infty} P(x) e^{i k x} d x
\end{aligned}
$$


are the Fourier transforms of $p_{n}(x)$ and $P(x)$. Disease density profiles for $R_{0} \neq 1$ are obtained by rescaling the solutions to equation 7 by a factor $R_{0}^{n}$. For illustrative purposes consider the case where $P(x)=(1 / 2)\left(x_{0}^{1 / 2} /\left(x_{0}+x\right)^{3 / 2}\right)$ which has a $-3 / 2$ inverse power law tail. In this case,

$$
\hat{P}(k)=1+\sqrt{2 \pi x_{0} k}\left[\sin \left(k x_{0}\right)\left(\frac{1}{2}-C\left(\sqrt{\frac{2}{\pi} k x_{0}}\right)\right)-\cos \left(k x_{0}\right)\left(\frac{1}{2}-S\left(\sqrt{\frac{2}{\pi} k x_{0}}\right)\right)\right]
$$

$$
\mathrm{x} / \mathrm{h}_{\mathrm{c}}=0.38
$$

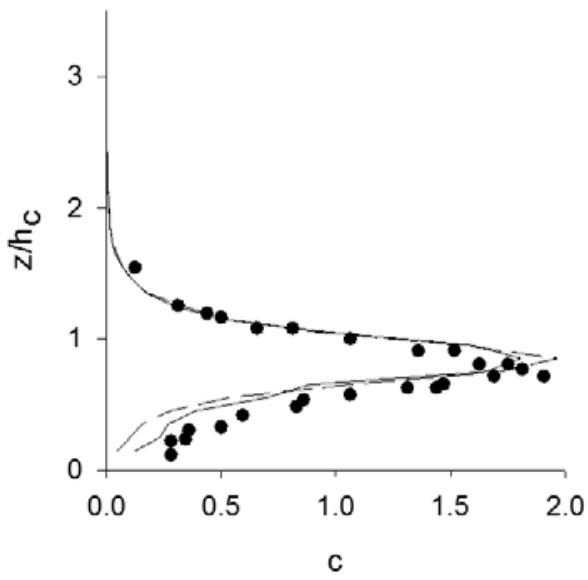

$\mathrm{x} / \mathrm{h}_{\mathrm{c}}=0.38$
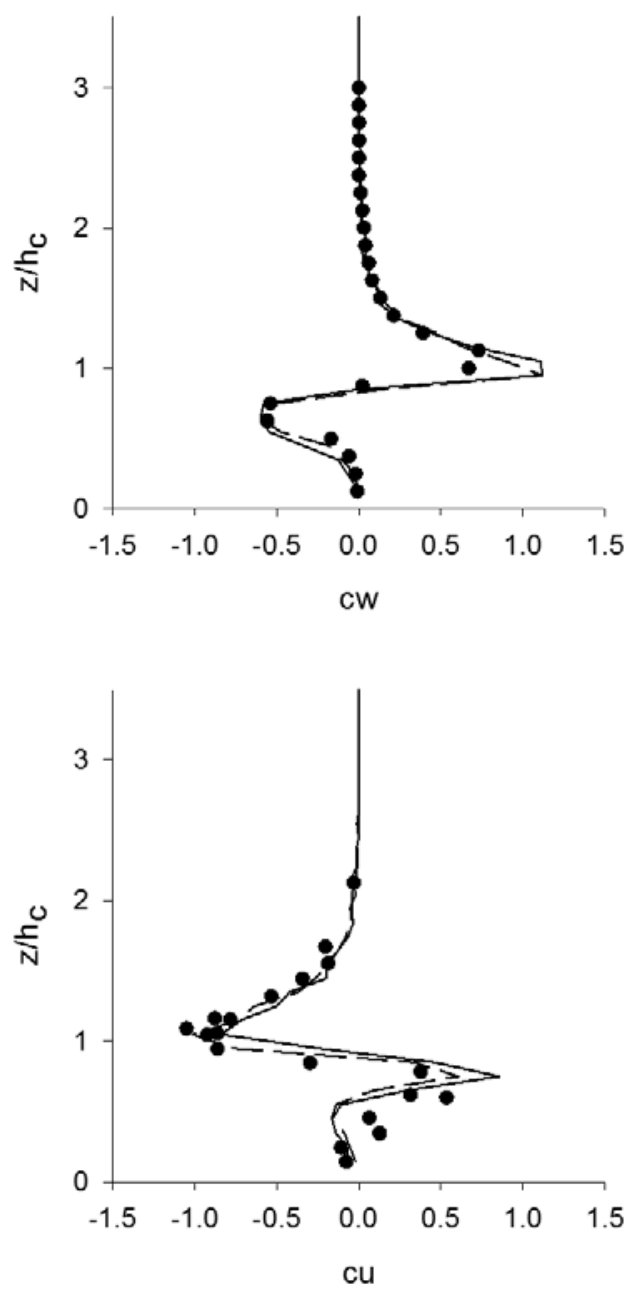

$\mathrm{x} / \mathrm{h}_{\mathrm{c}}=11.6$
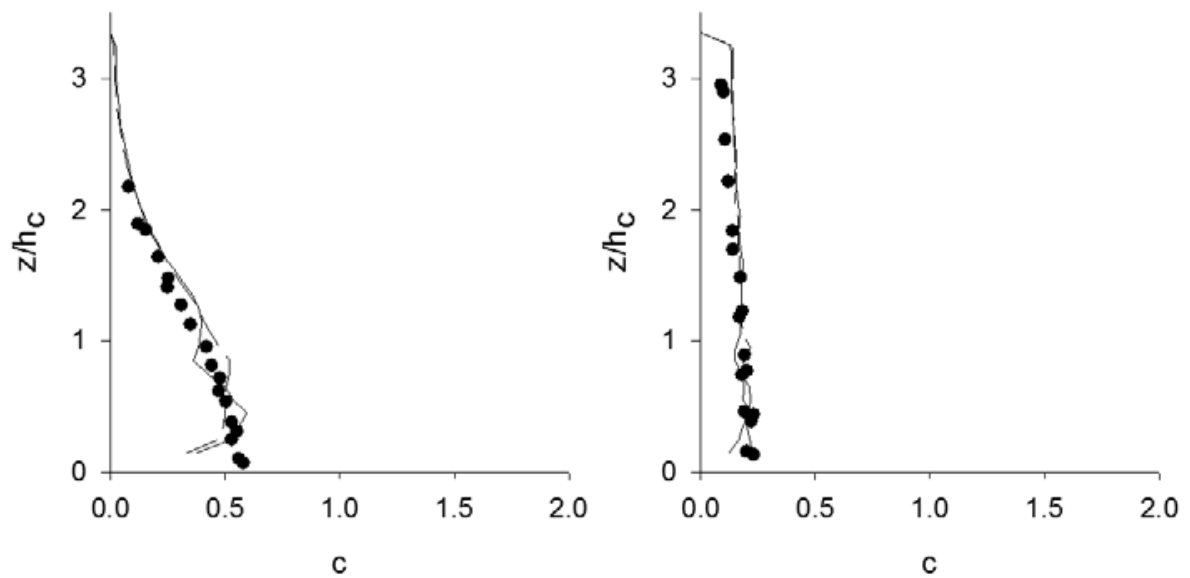

$\mathrm{x} / \mathrm{h}_{\mathrm{c}}=2.78$
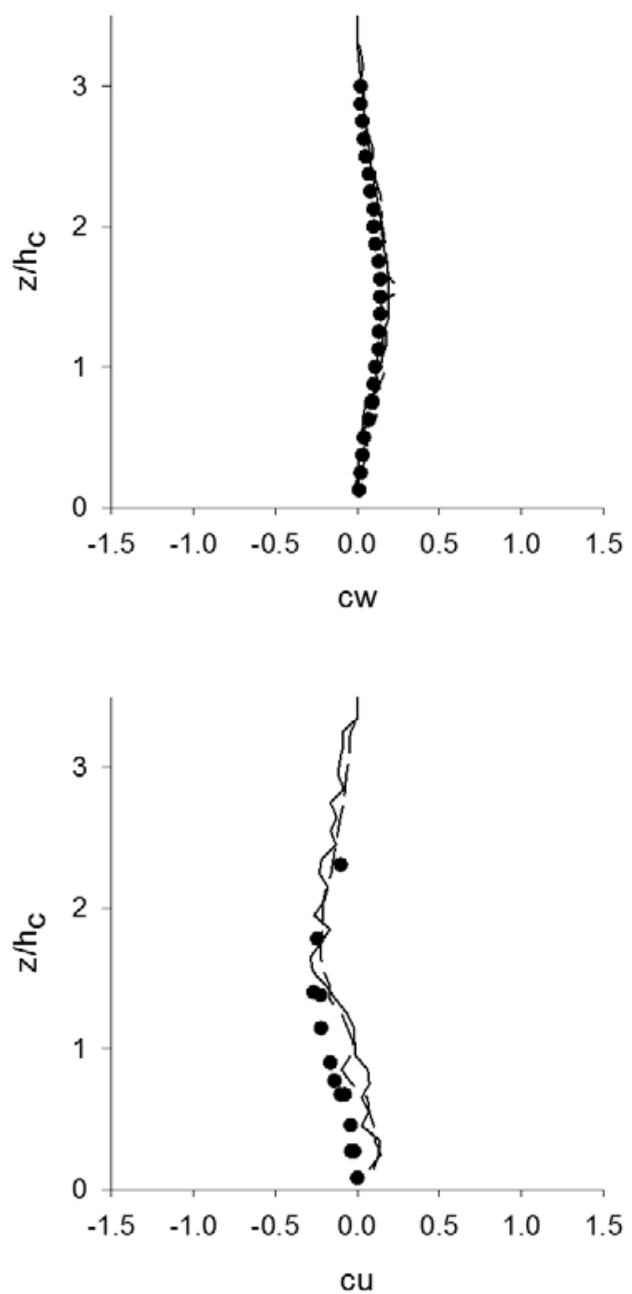

Fig. 2. Comparison of the measured (23) and predicted (non-Gaussian model, solid lines; Gaussian model, dashed lines) mean scalar concentrations, $c$, and mean scalar fluxes, $c u$ and $c w$, in the streamwise and vertical directions at three locations $x / h_{c}=0.38,2.78$, and 11.6 downwind of a line source located within a plant canopy of height $h_{c}$ at a height $0.85 h_{c}$. 
where $C\left(\sqrt{(2 / \pi) k x_{0}}\right)$ and $S\left(\sqrt{(2 / \pi) k x_{0}}\right)$ are Fresnel integrals. The behavior of $\hat{P}(k)$ at large distances is obtained in the limit $x_{0} k \rightarrow 0$. As $x_{0} k \rightarrow 0, \quad \hat{P}(k) \rightarrow 1-\sqrt{\pi k x_{0} / 2}$. Inserting the long-range asymptotic, $\hat{P}(k)=1-\sqrt{\pi k x_{0} / 2}$, into equation 7 gives $\hat{p}_{n}(k)=\left(1-\sqrt{\pi k x_{0} / 2}\right)^{n}$ when $\hat{p}_{0}(k)=1$, i.e., when the initial disease outbreak is localized around the origin $\left(p_{0}(x)=\delta(x)\right.$, where $\delta(x)$ is a Dirac delta function). Thus, $\hat{p}_{n+1}(k)-\hat{p}_{n}(k)=-\sqrt{\left(\pi k x_{0} / 2\right)} \hat{p}_{n}(k)$. Transforming back into real space and then taking the continuum limit (i.e., replacing the generation number, $n$, by time, $t$ and then replacing $\hat{p}_{t+1}(x)-\hat{p}_{t}(x)$ by $(\partial p / \partial t) T$, where $T$ is the time span between successive generations) gives a space-fractional diffusion equation of order $1 / 2$ :

$$
\frac{\partial p}{\partial t}=\frac{1}{T} \sqrt{\frac{\pi x_{0}}{2}} \frac{\partial^{1 / 2} p}{\partial x^{1 / 2}}
$$

Here as elsewhere and consistent with the results of ordinary calculus, the $n$th order fractional derivative of $p_{n}(x),\left(d^{n} p_{n} / d x^{n}\right)$, is defined by its Fourier transform: $(i k)^{n} \hat{p}_{n}(k)$.

An analogous calculation shows that contact distributions with heavy inverse power-law tails, $P(x) \sim x^{-\mu}$, give rise to $\mu$ th-order space-fractional diffusion equation when $1<\mu<3$ and that contact distributions with exponential tails, $P(x)=\lambda^{-1} e^{-x / \lambda}$, result in ordinary diffusion equations $(\partial p / \partial t)=\left(\lambda^{2} / T\right)\left(\partial^{2} p / \partial x^{2}\right)$ with diffusivity $D=\lambda^{2} T^{-1}$.

Spores within plant canopy turbulence undergo Lévy flights. Lévy flights, named after the French mathematician Paul Lévy, arose in a purely mathematical context in the first half of the last century (25). They comprise clusters of short step flights with longer movements between them. This pattern is repeated across all scales with the resultant clusters creating fractal patterns that have no characteristic scale and such that the distribution of move lengths has an inverse power-law tail, $p_{l}(l) \sim l^{-\mu}$ where $1<\mu<3$. The heavy-tailed contact distribution that characterizes model predictions for spore dispersal within plant canopy turbulence is indicative of Lévy flights with $\mu \approx 1$.

\section{DISCUSSION}

Ejections (updrafts) and sweeps (downdrafts) make the dominant contribution to turbulent transport into and out of plant canopies (16,35). These intermittent (non-Gaussian) turbulent flow structures also liberate the spores of important pathogens including Puccinia triticina (brown rust), P. striiformis (yellow rust), $P$. graminis (wheat stem rust), powdery mildews like Blumeria graminis (wheat powdery mildew), Peronospora tabacina (tobacco blue mold), and cucurbit mildews from host plant surfaces (1). And they are responsible for the lift-off minute aerially dispersed pests such as coccids and mites, and for seed abscission $(40,44)$. By carrying these organisms well above the canopy, ejections facilitate long-distance dispersal (32), a process that can have important consequences for the structuring of plant communities (31). It is therefore not surprising to find that several attempts have been made to incorporate ejections and sweeps into LS models of particle-trajectories in plant canopy turbulence $(17,36)$. These attempts have not been wholly successful. The models of Flesch and Wilson (17) and Reynolds (36) have a highly nonlinear dependency on velocity are so are incompatible with the Navier Stokes equations, the governing equations for fluid turbulence, and model agreement with experiment is worse or no better than that obtained with Thomson's Gaussian LS model (41). Here by drawing on the work of Franzese et al. (18) we showed how this long-standing difficulty with the formulation of LS models for dispersal in plant canopy turbulence can be overcome. Using this approach we devised a two-dimensional LS model that takes explicit account of sweeps and ejections and is compatible with the Navier Stokes equations. The new LS model was shown to predict accurately mean concentrations and turbulent fluxes of heat released as a passive scalar from an ex- tended line source within a model canopy in carefully controlled wind tunnel experiments (23). The three-dimensional form of this new non-Gaussian model is readily constructed and this would facilitate simulations of scalar dispersal from localized sources.

Equally good predictions were obtained with Thomson's Gaussian model. The utilitarian value of Thomson's model was not obvious a priori as it significantly underestimates the frequency of occurrence of high-velocity ejections and sweeps. Indeed, others have shown that predictions for dispersion in convective boundary-layers (a simpler flow than plant canopy turbulence) depends sensitivity on the parameterization of velocity skewness and kurtosis statistics which determine the frequency of occurrence of updrafts and downdrafts $(13,45)$. The finding is of considerable practical interest because Thomson's model is widely used in phytopathology (3-5) and is relatively easy to parameterize. The close correspondence between the predictions obtained with the new model and with Thomson's model suggests, in accordance with the speculations of Flesch and Wilson (17), that 'skewness and kurtosis statistics have a minimal effect [on predictions for dispersion within plant canopy turbulence] compared with other factors such as the strong gradients in velocity variance and shear stress'. Nonetheless, because Thomson's model is founded on Gaussian turbulent velocity statistics it cannot be coupled directly with realistic non-Gaussian models for the removal of passively released pathogenic spores and pollens from plant surfaces, and with realistic non-Gaussian models of seed abscission $(1,40)$. The number of high-velocity ejections (with speeds $>4 \mathrm{~ms}^{-1}$ ) within the lower half of the model canopy of Raupach et al. (34), is for example, underestimated by a factor of about 6 when turbulent velocity fluctuations are taken to be Gaussian. Much better predicted are the number of ejections with speeds $>1 \mathrm{~ms}^{-1}$.

Model predictions for contact distributions for fungal spores (as well as minute aerially dispersed pests, seeds and pollens) were found to have heavy inverse power-law tails at the field scale. Heavy-tailed contact distributions are also produced by Thomson's model but without the confirmation that the relatively high frequency of occurrence of ejections and sweeps can be safely neglected, this prediction would have been questionable and could have been attributed to inappropriate model formulation. The model prediction is expected to hold true irrespective of buoyancy because "one consequence of the mixing-layer analogy is that the turbulent eddy structure near the top of the

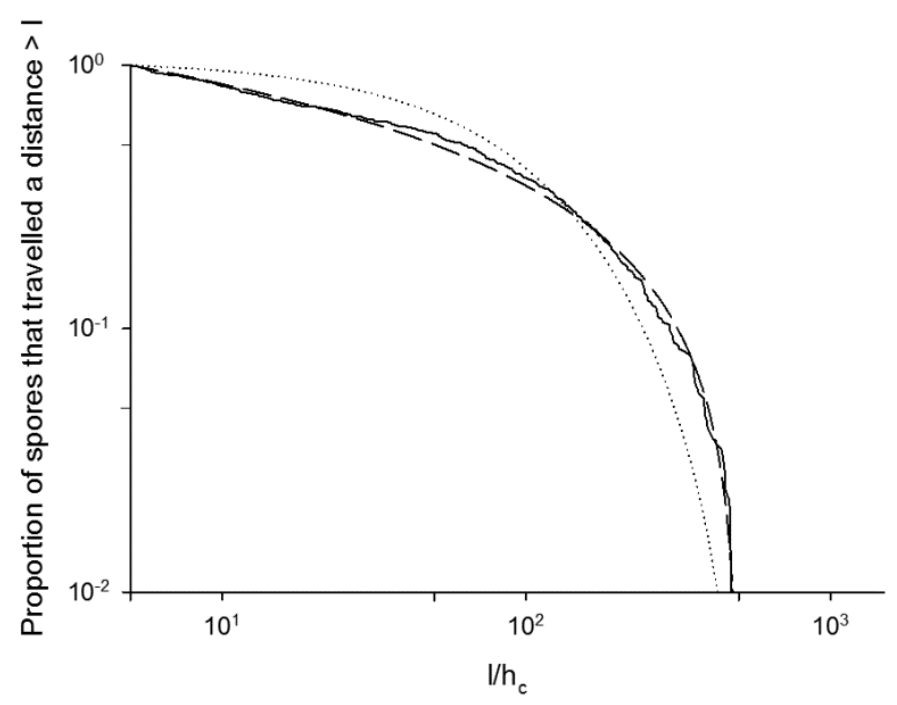

Fig. 3. Proportion of spores that are predicted to travel a distance, $l$, after first exiting a plant canopy of height $h_{c}$ and before first reentering it (solid line). Shown for comparison is the best fit power-law (dashed line) and the best-fit exponential (dotted line). 
canopy has a similar qualitative behavior (though not identical quantitative properties) across a wide range of buoyancy conditions" (35). Heavy-tailed distributions albeit with a larger scaling exponent, -2 , may also characterize spore dispersal within convective atmospheric boundary-layers with wind shear (37). Consequently, wind-dispersed pathogens are predicted to cause accelerating waves of plant disease over a vast range of scales, from the field scale through to continental scales. These predictions are supported by recent analyses of field data for wheat stripe rust and historical plant disease epidemics (potato late blight, wheat stem rust, and southern corn leaf blight) at the continental scale $(5,12,30)$.

It was also shown that plant disease epidemics and infestations resulting from heavy-tailed contact distributions are governed by fractional diffusion equations and by Lévy flights. Exponentialtailed contact distributions give rise to ordinary diffusion equations. Fractional diffusion equations and Lévy flights have now found numerous applications outside of phytopathology (28). It would be interesting to see whether this understanding can, as with the study of human disease epidemics $(7-9,19,20)$, be imported into the study of plant disease epidemics. Brockmann et al. (9) and González et al. (19) uncovered Lévy flight characteristics in patterns of human mobility that were later shown to have important consequences for quantifying the spread of viruses and for the development of models of human disease epidemics $(8,20)$. Following Hanert et al. (20), new models of plant disease epidemics that account for heavy-tailed fungal spore dispersal could be obtained from current reaction-diffusion models by replacing the diffusion term with a fractional diffusion term. Fractional diffusion leads to an exponential acceleration of the epidemic's front, a power-law decay of the front's leading tail and to clustering of new foci of infection (28). These predictions could be compared quantitatively with observations $(12,30)$ so that the models can be refined and expanded thereby making them as general as possible. The models could then be used to examine the potential effectiveness of detection and control strategies that strive to reduce or eradicate disease outbreaks whilst minimizing costs. This effectiveness could also be examined in numerical simulations using synthetic Lévy flights. Lévy flights as vectors of disease have only recently appeared in the literature on phytopathology (14) and then without much justification because such movement patterns have not been observed directly in disease-carrying organisms, despite their widespread occurrence amongst predators (43). Nonetheless, the development of citrus variegated chlorosis does appear to be consistent with the vector of disease, suctorial sharp-shooter leafhoppers (Hemiptera: Cicadellidae), having Lévy flights movement patterns (27). Missing from the study of Dybiec et al. (43) was the realization that fungal spores within plant canopy turbulence undergo Lévy flights.

\section{ACKNOWLEDGMENTS}

This research is funded by the Biotechnology and Biological Sciences Research Council (BBSRC). I thank D. Reynolds for bringing to my attention the literature on wingless arthropod dispersal.

\section{LITERATURE CITED}

1. Aylor, D. E. 1990. The role of intermittent wind in the dispersal of fungal pathogens. Annu. Rev. Phytopathol. 28:73-92.

2. Aylor, D. E. 1999. Biophysical scaling and the passive dispersal of fungus spores: Relationship to integrated pest management strategies. Agric. For. Meteorol. 97:275-292.

3. Aylor, D. E., and Flesch, T. K. 2001. Estimating spore release rates using a Lagrangian stochastic simulation model. J. Appl. Meteorol. 40:11961208.

4. Aylor, D. E., Fry, W. E., Mayton, H., and Andrade-Piedra, J. L. 2001. Quantifying the rate of release and escape of Phytophthora infestans sporangia from a potato canopy. Phytopathology 91:1189-1196.

5. Aylor, D. E., Schmale, D. G., III, Shields, E. J., Newcomb, M., and
Nappo, C. J. 2011. Tracking the potato late blight in the atmosphere using unmanned aerial vehicles and Lagrangian modelling. Agric. For. Meteorol. 151:251-260.

6. Borgas, M. S., and Yeung, P. K. 1998. Conditional fluid-particle accelerations in turbulence. Theor. Comp. Fluid Dynamics 11:69-93.

7. Brockmann, D. 2009. Human mobility and spatial disease dynamics. Pages 55-81 in: Reviews of Nonlinear Dynamics and Complexity. H. G. Schuster, ed. Wiley-VCH Verlag GmbH \& Co. KGaA, Weinheim, Germany.

8. Brockmann, D., and Hufnagel, L. 2007. Front propagation in reactionsuperdiffusion dynamics: Taming Lévy flights with fluctuations. Phys. Rev. Lett. 98:178301.

9. Brockmann, D., Hufnagel, L., and Geisel, T. 2005. The scaling laws of human travel. Nature 439:462-465.

10. Cannas, S. A., Marco, D. E., and Montemurro, M. A. 2006. Long range dispersal and spatial pattern formation in biological invasions. Math. Biosci. 203:155-170.

11. Clark, J. S., Lewis, M., and Horvath, L. 2001. Invasion by extremes: Population spread with variation in dispersal and reproduction. Am. Nat. 157:537-554.

12. Cowger, C., Wallace, L. D., and Mundt, C. C. 2005. Velocity of spread of wheat stripe rust epidemics. Phytopathology 95:972-832.

13. Du, S. M., Wilson, J. D., and Yee, E. 1994. Probability density-functions for velocity in the convective boundary-layer, and implied trajectory models. Atmos. Environ. 28:1211-1217.

14. Dybiec, B., Kleczkowski, A., and Gilligan, C. A. 2009. Modelling control of epidemics spreading by long-range interactions. J. Roy. Soc. Interface 6:941-950.

15. Ferrandino, F. J. 1993. Dispersive epidemic waves. I. Focus expansion within a linear planting. Phytopathology 83:795-802.

16. Finnigan, J. 2000. Turbulence in plant canopies. Annu. Rev. Fluid Mech. 32:519-571.

17. Flesch, T. K., and Wilson, J. D. 1992. A two-dimensional trajectorysimulation model for non-Gaussian inhomogeneous turbulence within plant canopies. Boundary-Layer Meteorol. 61:349-374.

18. Franzese, P., Luhar, A. K., and Borgas, M. S. 1999. An efficient Lagrangian stochastic model of vertical dispersion in the convective boundary layer. Atmos. Environ. 33:2337-2345.

19. González, M. C., Hidalgo, C. A., and Barabási, A.-L. 2008. Understanding individual human mobility patterns. Nature 453:779-782.

20. Hanert, E., Schumacher, E., and Deleersnijder, E. 2011. Front dynamics in fractional-order epidemic models. J. Theor. Biol. 279:9-16.

21. Hastings, A., Cuddington, K., Davis, K. F., Dugaw, C. J., Elmendorf, S., Freestone, A., Harrison, S., Holland, M., Lambrinos, J., Malvadkar, U., Melbourne, B. A., Moore, K., Taylor, C., and Thomson, D. 2005. The spatial spread of invasions: new developments in theory and evidence. Ecol. Letts. 8:91-101.

22. Kot, M., Lewis, M. A., and van den Driessche, P. 1996. Dispersal data and the spread of invading organisms. Ecology 77:2027-2042.

23. Legg, B. J., Raupach, M. R., and Coppin, P. A. 1986. Experiments on scalar dispersion within a model plant canopy part III: An elevated line source. Boundary-Layer Meteorol. 35:277-302.

24. Lett, C., and Østergård, H. 2000. A stochastic model simulating the spatiotemporal dynamics of yellow rust on wheat. Acta Phytopathol. Entomol. Hung. 35:287-293.

25. Lévy, P. 1937. Théorie de l'addition des variables aléatoires. (Monographies des Probabilités, publiés sous la direction de E. Borel, no. 1.) Guathier Villars, Paris.

26. Madden, L. V., Hughes, G., and van den Bosch, F. 2006. The Study of Plant Disease Epidemics. American Phytopathological Society, St. Paul, MN.

27. Martins, M. L., Ceotto, G., Alves, S. G., Bufon, C. C. B. and Silva, J. M. 2000. Cellular automata model for citrus variegated chlorosis. Phys. Rev. E 62:7024-7030.

28. Metzler, R., and Klafter, J. 2004. The restaurant at the end of the random walk: recent developments in the description of anomalous transport by fractional dynamics. J. Phys. A 37:R161-208.

29. Mollison, D. 1977. Spatial contact models for ecological and epidemic spread. J. Roy. Stat. Soc. B 39:283-326.

30. Mundt, C. C., Sackett, K. E., Wallace, L. D., Cowger, C., and Dudley, J. P. 2009. Long-distance dispersal and accelerating waves of disease: Empirical relationships. Am. Nat. 173:456-466.

31. Nathan, R. 2006. Long-distance dispersal of plants. Science 313:786-788.

32. Nathan, R., Katul, G. G., Horn, H. S., Thomas, S. M., Oren, R., Avissar, R., Pacala, S. W., Levin, S. A. 2002. Mechanisms of long-distance dispersal of seeds by wind. Nature 418:409-413.

33. Pope, S. B. 1987. Consistency conditions for random-walk models of turbulent dispersion. Phys. Fluids 30:2374-2379.

34. Raupach, M. R., Coppin, P. A., and Legg, B. J. 1986. Experiments on scalar dispersion within a model plant canopy part I: The turbulent 
structure. Boundary-Layer Meteorol. 35:21-52.

35. Raupach, M. R., Finnigan, J. J., and Brunet, Y. 1996. Coherent eddies and turbulence in vegetation canopies: The mixing-layer analogy. BoundaryLayer Meteorol. 78:351-382.

36. Reynolds, A. M. 1998. On the formulation of Lagrangian stochastic models for scalar dispersion within plant canopies. Boundary-Layer Meteorol. 86:333-344.

37. Reynolds, A. M. 2011. Exponential and power-law contact distributions represent different atmospheric conditions. Phytopathology 101:1465-1470.

38. Shaw, M. W. 1995. Simulation of population expansion and spatial pattern when individual dispersal distributions do not decline exponentially with distance. Proc. Roy. Soc. B 259:243-248.

39. Shaw, M. W., Harwood, T. D., Wilkinson, M. J., and Elliott, L. 2006. Assembling spatially explicit landscape models of pollen and spore dispersal by wind for risk assessment. Proc. Roy. Soc. B 273:1705-1713.

40. Soons, M. B., and Bullock, J. M. 2008. Non-random seed abscission, longdistance wind dispersal and plant migration rates. J. Ecol. 96:581-590.

41. Thomson, D. J. 1987. Criteria for the selection of stochastic models of particle trajectories in turbulent flows. J. Fluid Mech. 180:529-556.
42. Thomson, D. J., and Montgomery, M. R. 1994. Reflection boundary conditions for random walk models of dispersion in non-gaussian turbulence. Atmos. Environ. 28:1981-1987.

43. Viswanathan, G. M., da Luz, M. G. E., Raposo, E. P., and Stanley, H. E. 2011. The Physics of Foraging: An Introduction to Random Searches and Biological Encounters. Cambridge University Press, Cambridge, UK

44. Washburn, J. O., and Washburn, L. 1984. Active aerial dispersal of minute wingless arthropods: Exploitation of boundary-layer velocity gradients. Science 223:1088-1089.

45. Weil, J. C. 1990. A diagnosis of the asymmetry in top-down and bottomup diffusion using a Lagrangian stochastic model. J. Atmos. Sci. 47:501515.

46. White, E. P., Enquist, B. J., and Green, J. L. 2008. On estimating the exponent of power-law frequency distributions. Ecology 89:905-912.

47. Wingen, L. U., Brown, J. K. M., and Shaw, M. W. 2007. The population genetic structure of clonal organisms generated by exponentially bounded and fat-tailed dispersal. Genetics 177:435-448.

48. Zadoks, J. C. 2001. Plant disease epidemiology in the twentieth century: A picture by means of selected controversies. Plant Dis. 85:808-816. 